\title{
Heisenberg Uncertainty Relations as Statistical Invariants
}

\author{
Aniello Fedullo \\ Department of Physics "E. R. Caianiello", University of Salerno, Italy \\ afedullo@unisa.it
}

«Surely, one would like to be able to deduce the quantitative laws of quantum mechanics directly from their anschaulich foundations, that is, essentially, relation $\delta \mathrm{p} \delta \mathrm{q} \sim \mathrm{h} »$.

Werner Heisenberg [8, p. 196]

\begin{abstract}
For a simple set of observables we can express, in terms of transition probabilities alone, the Heisenberg Uncertainty Relations, so that they are proven to be not only necessary, but sufficient too, in order for the given observables to admit a quantum model. Furthermore distinguished characterizations of strictly complex and real quantum models, with some ancillary results, are presented and discussed.
\end{abstract}

\section{Foreword}

The problem of understanding the empirical basis of the quantum mechanical formalism has been approached, starting from eighties, by means of the method of statistical invariants [1] [2]. The main idea of this approach, borrowed from the Klein's program of Erlangen [3], is to classify the probabilistic models according to statistical invariants, expressed in terms of the transition probabilities of the physical observables. That is to say, one considers the transition probabilities as the basic empirical data from which the mathematical model should be deduced. The statistical invariants for some simple systems were explicitly computed and it was shown that they allowed to distinguish among Kolmogorovian, real Hilbert space and complex Hilbert space models. Actually necessary and sufficient conditions for the existence of each model were found [1] [4] [5]. When applied to the quantum-mechanical transition probabilities, they proved not only the necessity of a non classical probabilistic model, but also the necessity of using complex rather than real Hilbert spaces [1] [5], so offering the solution to the open problem of "...singling out in full generality the empirical basis for the choice of complex numbers in quantum theory....'[6]. Furthermore the Kolmogorovian statistical invariant was recognized as a form of the celebrated Bell inequality [1], expressed by the transition probabilities instead of the correlation functions. In the present paper we pursue the study of a triple of two-dimensional observables undertaken 
in [1] and, by means of the notion of quantum models, we show as their statistical invariants represent the Heisenberg uncertainty relations expressed in terms of transition probabilities alone. This allow us to affirm that uncertainty relations are not only necessary, but sufficient too, in order for the given observables to admit a quantum model.

\section{Preliminary definitions and results}

In the oft quoted paper [1] a triple $A, B, C$ of two-valued observables subject to take values $\left(a_{\alpha}\right),\left(b_{\beta}\right),\left(c_{\gamma}\right)$ were studied, given their transition probabilities

$$
P\left(A=a_{\alpha} \mid B=b_{\beta}\right), P\left(B=b_{\beta} \mid C=c_{\gamma}\right), P\left(C=c_{\gamma} \mid A=a_{\alpha}\right)
$$

under the symmetry assumptions

$$
P\left(A=a_{\alpha} \mid B=b_{\beta}\right)=P\left(B=b_{\beta} \mid A=a_{\alpha}\right), . .1
$$

For observable is meant any quantity arising from experiments of whatever nature. The transition probability $P\left(A=a_{\alpha} \mid B=b_{\beta}\right)$ is the conditional probability that $A$ takes the value $a_{\alpha}$ conditioned by the fact that $B$ is known to assume the value $b_{\beta}$. We will denote the transition probabilities (1) as

$$
\begin{aligned}
& P(A \mid B)=\left[\begin{array}{cc}
p & 1-p \\
1-p & p
\end{array}\right]=\left[\begin{array}{ll}
\cos ^{2} \frac{\alpha}{2} & \sin ^{2} \frac{\alpha}{2} \\
\sin ^{2} \frac{\alpha}{2} & \cos ^{2} \frac{\alpha}{2}
\end{array}\right], \\
& P(B \mid C)=\left[\begin{array}{cc}
q & 1-q \\
1-q & q
\end{array}\right]=\left[\begin{array}{ll}
\cos ^{2} \frac{\beta}{2} & \sin ^{2} \frac{\beta}{2} \\
\sin ^{2} \frac{\beta}{2} & \cos ^{2} \frac{\beta}{2}
\end{array}\right], \\
& P(C \mid A)=\left[\begin{array}{cc}
r & 1-r \\
1-r & r
\end{array}\right]=\left[\begin{array}{ll}
\cos ^{2} \frac{\gamma}{2} & \sin ^{2} \frac{\gamma}{2} \\
\sin ^{2} \frac{\gamma}{2} & \cos ^{2} \frac{\gamma}{2}
\end{array}\right],
\end{aligned}
$$

assuming, unless otherwise specified, that

$$
0<p, q, r<1
$$

for which the angles can be chosen such that

$$
0<\alpha, \beta, \gamma<\pi
$$

The transition probabilities (1) were said to admit a complex (resp. a real) Hilbert space model if there exist three orthonormal bases $\left\{\phi_{\alpha}\right\},\left\{\psi_{\beta}\right\},\left\{\chi_{\gamma}\right\}$ of a twodimensional complex (resp. real) Hilbert space $\mathcal{H}$ such that

$$
P\left(A=a_{\alpha} \mid B=b_{\beta}\right)=\left|\left\langle\phi_{\alpha} \mid \psi_{\beta}\right\rangle\right|^{2}, \ldots
$$

\footnotetext{
${ }^{1}$ Here and in the following ellipsis stands for all similar relations involving the observables left over.
} 
In particular a complex Hilbert space model was said a spin model if the three o. $\mathrm{n}$. bases can be taken as the normalized eigenvectors $\psi_{\alpha}\left(u_{A}\right), \psi_{\beta}\left(u_{B}\right), \psi_{\gamma}\left(u_{C}\right)$ $(\alpha, \beta, \gamma=1,2)$ of the spin operators $u_{A} \cdot \sigma, u_{B} \cdot \sigma, u_{C} \cdot \sigma$ for some $u_{A}, u_{B}, u_{C} \in$ $S^{(2)}$, where $S^{(2)}$ denotes the real unit sphere in $\mathbb{R}^{3}$ and $u \cdot \sigma$ is defined in terms of the Pauli matrices

$$
\sigma_{1}:=\left[\begin{array}{cc}
0 & 1 \\
1 & 0
\end{array}\right], \quad \sigma_{2}:=\left[\begin{array}{cc}
0 & -i \\
i & 0
\end{array}\right], \quad \sigma_{3}:=\left[\begin{array}{cc}
1 & 0 \\
0 & -1
\end{array}\right]
$$

as

$$
u \cdot \sigma:=u_{1} \sigma_{1}+u_{2} \sigma_{2}+u_{3} \sigma_{3}
$$

In the latter case we can write (8), in terms of the angles $\widehat{u_{A} u_{B}}, \widehat{u_{B} u_{C}}, \widehat{u_{C} u_{A}}$, as

$$
\begin{aligned}
& \left|\left\langle\psi_{1}\left(u_{A}\right) \mid \psi_{1}\left(u_{B}\right)\right\rangle\right|^{2}=\cos ^{2} \frac{\widehat{u_{A} u_{B}}}{2}, \ldots \\
& \left|\left\langle\psi_{1}\left(u_{A}\right) \mid \psi_{2}\left(u_{B}\right)\right\rangle\right|^{2}=\sin ^{2} \frac{\widehat{u_{A} u_{B}}}{2}, \ldots
\end{aligned}
$$

In the present paper we prefer focus our attention on observables rather than transition probabilities alone. So we can recover the usual formalism of quantum mechanics [7] according to observables are postulated in correspondence with self-adjoint operators on a suitable complex Hilbert space. In this frame uncertainty relations we are concerned found their most general formulation, so that we are led to give the following

Definition 2.1. The observables $A, B, C$ are said to admit a quantum model if and only if there exists a complex Hilbert space $\mathcal{H}$ and self-adjoint operators $\hat{A}, \hat{B}, \hat{C}$ acting 2 on it such that the values of each observable coincide with the eigenvalues of the corresponding operator and the transition probabilities (1) admit the complex Hilbert space model defined by the o. $n$. bases of $\mathcal{H}$ made up of the normalized eigenvectors 3 of $\hat{A}, \hat{B}, \hat{C}$. The model will be called a real quantum model if $\mathcal{H}$ can be taken real, or a strictly complex quantum model otherwise.

Next theorem shows a first expected link between quantum models of observables and Hilbert space models of the relative transition probabilities.

Theorem 2.1. The following assertions are equivalent: i) the observables $A, B, C$ admit a quantum model; ii) their transition probabilities admit a spin model; iii) their transition probabilities admit a complex Hilbert space model.

\footnotetext{
${ }^{2} \hat{A}, \hat{B}, \hat{C}$ are defined up to a common unitary transformation, cfr. [1] corollary 8. A self-adjoint operator having eigenvalues $\mp 1$ is just a spin operator.

${ }^{3}$ Said eigenstates as well.
} 
Proof. To prove that i) is equivalent to ii) it is suffices to observe that, for every observable $X$ (with values $x_{1}, x_{2}$ ) of the triple $A, B, C$, the operator

$$
\hat{S}_{X}:=\frac{2}{x_{1}-x_{2}} \hat{X}-\frac{x_{1}+x_{2}}{x_{1}-x_{2}} \hat{1}
$$

where $\hat{1}$ is the identity operator, has the eigenvalues $\mp 1$, so it is a spin operator and, due to $\left[\hat{S}_{X}, \hat{X}\right]=0$, it has the same eigenvectors of $\hat{X}$. Further, the equivalence between ii) and iii) was proven in [1], theorem -4 .

Remark 2.1. The particular case of real quantum models will be discussed later. Notice that the precedent result deals with a (linear) rescaling of the observables preserving probabilities. Further observe that the spin operators $\hat{S}_{A}, \hat{S}_{B}, \hat{S}_{C}$ can be written as $u_{A} \cdot \sigma, u_{B} \cdot \sigma, u_{C} \cdot \sigma$ respectively, for suitable unit vectors 5 $u_{A}, u_{B}, u_{C} \in S^{(2)}$, which so remain associated to the self-adjoint operators $\hat{A}, \hat{B}, \hat{C}$ respectively.

In this framework we are able to reformulate some results of [1] as follows:

Theorem 2.2. The following assertions are equivalent: i) the observables $A, B, C$ admit a quantum model; ii) there exist $u_{A}, u_{B}, u_{C} \in S^{(2)}$ such that $u_{A} \cdot u_{B}=$ $\cos \alpha, u_{B} \cdot u_{C}=\cos \beta, u_{C} \cdot u_{A}=\cos \gamma$; iii) $1-\cos ^{2} \alpha-\cos ^{2} \beta-\cos ^{2} \gamma+$ $2 \cos \alpha \cos \beta \cos \gamma \geq 0$. Moreover inequalities iii) is saturated if and only if $A, B, C$ admit a real quantum model; in such a case, and only then, the unit vectors in ii) are coplanar.

Proof. By theorem 2.1, i) is equivalent to the existence of a spin model for the transition probabilities; this in turn, by eqs. (8), (11) and (12), as well as due to $\cos \widehat{u_{A} u_{B}}=u_{A} \cdot u_{B}, \cos \widehat{u_{B} u_{C}}=u_{B} \cdot u_{C}, \widehat{u_{A} u_{C}}=u_{A} \cdot u_{C}$, is easily recognized equivalent to ii); the equivalence between ii) and iii) was established in [1] ${ }^{4}$, proposition 3, just as, from theorems 9 and 10 therein, it follows straight the last statement to be proven.

Remark 2.2. The inequality in iii) of the precedent theorem is said a statistical invariant [2] for a quantum model. It is said in particular a statistical invariant for a real quantum model if the inequality is saturated, or a statistical invariant for a strictly complex quantum model, otherwise. Many equivalent forms of these invariants were discovered in $[1]^{4}$ and some others, involving uncertainty relations, will appear below.

\footnotetext{
${ }^{4}$ Cfr. Appendix, 1 .

${ }^{5}$ Any common unitary transformation of $\hat{A}, \hat{B}, \hat{C}$, referred to in note 2 , induces a common rotation of $u_{A}, u_{B}, u_{C}$, cfr. [1], corollary 8 proof.

${ }^{6}$ Observe that $u_{A}, u_{B}, u_{C}$ are necessarily distinct and pairwise non collinear, due to the assumption (5).
} 


\section{Uncertainty Relations}

Uncertainty relations were introduced in quantum mechanics by Heisenberg [8] and successively extended and strengthen by many authors [10][11] In this paper we will refer to the following stronger form:

Theorem 3.1. (Schrödinger [11]) For every couple of self-adjoint operators $\hat{X}, \hat{Y}$ acting on a complex Hilbert space $\mathcal{H}$ and for every state $\psi$ the following inequality holds?:

$$
\operatorname{Var}(\hat{X}) \operatorname{Var}(\hat{Y}) \geq\left(\frac{1}{2}\langle\{\hat{X}, \hat{Y}\}\rangle-\langle\hat{X}\rangle\langle\hat{Y}\rangle\right)^{2}+\left(\frac{1}{2 i}\langle[\hat{X}, \hat{Y}]\rangle\right)^{2}
$$

where $\langle\hat{Z}\rangle:=\langle\psi|\hat{Z}| \psi\rangle$ and $\operatorname{Var}(\hat{Z}):=\left\langle\widehat{Z}^{2}\right\rangle-\langle\widehat{Z}\rangle^{2}$ are resp. the average of $\hat{Z}$ and the variance of $\hat{Z}$ in the state $\psi$, with $[\hat{X}, \hat{Y}]:=\hat{X} \hat{Y}-\hat{Y} \hat{X}$ and $\{\hat{X}, \hat{Y}\}:=\hat{X} \hat{Y}+\hat{Y} \hat{X}$ being the commutator, resp. the anticommutator, of $\hat{X}$ and $\hat{Y}$.

Proof. Cfr. for example reference [12] and, of course, [11].

Remark 3.1. In the following lemma 4.4 we show that, for the operators we consider in this paper, the inequality expressing the Heisenberg-Schrödinger uncertainty relation is in fact saturated, so that it assumes the form of an identity. From now on $\mathcal{H}$ will denote a two-dimensional complex Hilbert space.

\section{Some useful lemmas}

Lemma 4.1. Whichever $\hat{X}, \hat{Y}, \hat{Z}$ are taken among the operators $\hat{A}, \hat{B}, \hat{C}$ associated to the given observables $A, B, C$ one has, for every state,

$$
\langle\hat{Z}\rangle=\frac{z_{1}+z_{2}}{2}+\frac{z_{1}-z_{2}}{2}\left\langle u_{Z} \cdot \sigma\right\rangle
$$

and

$$
\operatorname{Var}(\hat{Z})=\left(\frac{z_{1}-z_{2}}{2}\right)^{2} \operatorname{Var}\left(u_{Z} \cdot \sigma\right)
$$

where $z_{1}, z_{2}$ are the values of the observable $Z$, as well as

$\frac{1}{2}\langle\{\hat{X}, \hat{Y}\}\rangle-\langle\hat{X}\rangle\langle\hat{Y}\rangle=\frac{x_{1}-x_{2}}{2} \frac{y_{1}-y_{2}}{2}\left(\frac{1}{2}\left\langle\left\{u_{X} \cdot \sigma, u_{Y} \cdot \sigma\right\}\right\rangle-\left\langle u_{X} \cdot \sigma\right\rangle\left\langle u_{Y} \cdot \sigma\right\rangle\right)$

\footnotetext{
${ }^{7}$ For a recent review cfr. [9] and the bibliography therein.

${ }^{8}$ As known a state is defined as a norm 1 element of $\mathcal{H}$ up to a phase factor.

${ }^{9}$ The first addend in the r. h. s. is said the covariance term and the second the commutator term.
} 
and

$$
\frac{1}{2 i}\langle[\hat{X}, \hat{Y}]\rangle=\frac{x_{1}-x_{2}}{2} \frac{y_{1}-y_{2}}{2} \frac{1}{2 i}\left\langle\left[u_{X} \cdot \sigma, u_{Y} \cdot \sigma\right]\right\rangle .
$$

Consequently the Heisenberg-Schrödinger uncertainty relation (14) holds for a couple of operators $\hat{X}, \hat{Y}$ acting on $\mathcal{H}$ if and only if it holds for the associated spin operators $u_{X} \cdot \sigma, u_{Y} \cdot \sigma$. Furthermore the former relation is saturated if and only if the latter is.

Proof. Cfr. Appendix, 2.

Lemma 4.2. For every $u, v, w \in S^{(2)}$ the following identities hold in each eigenstate $\psi_{k}(w)$ of $w \cdot \sigma(k=1,2)$ :

$$
\begin{gathered}
\langle u \cdot \sigma\rangle=(-1)^{k-1} u \cdot w, \\
\operatorname{Var}(u \cdot \sigma)=1-(u \cdot w)^{2}, \\
\frac{1}{2 i}\langle[u \cdot \sigma, v \cdot \sigma]\rangle=(u \times v) \cdot w
\end{gathered}
$$

and just for every state:

$$
\frac{1}{2}\langle\{u \cdot \sigma, v \cdot \sigma\}\rangle=u \cdot v
$$

Proof. Cfr. Appendix, 3

Lemma 4.3. For every state $\psi$ of the Hilbert space $\mathcal{H}$ there is a $w \in S^{(2)}$ such that $\psi=\psi_{1}(w)$, where $\psi_{1}(w)$ is the eigenstat 10 of the spin operator $w \cdot \sigma$, corresponding to the eigenvalue 1.

Proof. We can put, up to an non influential phase factor, $\psi=\left[\begin{array}{ll}\left|\psi_{1}\right| & \Re\left(\psi_{2}\right)+i \Im\left(\psi_{2}\right)\end{array}\right]^{T}$ while as known $11 \psi_{1}(w)=\left[\begin{array}{ll}\sqrt{\frac{1+w_{3}}{2}} & \frac{w_{1}+i w_{2}}{\sqrt{2\left(1+w_{3}\right)}}\end{array}\right]^{T}$, so that we can solve the vector equation $\psi_{1}(w)=\psi$ for $w$, getting $w_{1}=2\left|\psi_{1}\right| \Re\left(\psi_{2}\right), w_{2}=2\left|\psi_{1}\right| \Im\left(\psi_{2}\right)$, $w_{3}=2\left|\psi_{1}\right|^{2}-1$.

Lemma 4.4. For every couple of self-adjoint operators $\hat{X}, \hat{Y}$ on a two-dimensional complex Hilbert space $\mathcal{H}$ and for every state $\psi$ the following identity holds:

$$
\operatorname{Var}(\hat{X}) \operatorname{Var}(\hat{Y})=\left(\frac{1}{2}\langle\{\hat{X}, \hat{Y}\}\rangle-\langle\hat{X}\rangle\langle\hat{Y}\rangle\right)^{2}+\left(\frac{1}{2 i}\langle[\hat{X}, \hat{Y}]\rangle\right)^{2}
$$

\footnotetext{
${ }^{10}$ One has also that $\psi=\psi_{2}(-w)$, but it is not required here; $w$ is known as a representation of the state $\psi$ on the Bloch's sphere $S^{(2)}$.

${ }^{11}$ In a basis of $\mathcal{H}$ in which the Pauli matrices have the usual form (9), cfr. for example [1], p. 170 .
} 
Proof. Due to lemma 4.1 we can limit ourselves to the case in which $\hat{X}, \hat{Y}$ are spin operators, that is $\hat{X}=u \cdot \sigma$ and $\hat{Y}=v \cdot \sigma$ for suitable $u, v \in S^{(2)}$. However the generic state $\psi$, by lemma 4.3 , can be written as $\psi=\psi_{1}(w)$, for a suitable $w \in S^{(2)}$. So (23) by means of lemma 4.2 can be written as

$$
\left(1-(u \cdot w)^{2}\right)\left(1-(v \cdot w)^{2}\right)=(u \cdot v-(u \cdot w)(v \cdot w))^{2}+((u \times v) \cdot w)^{2} ;
$$

expanding and simplifying it becomes

$$
1-(u \cdot w)^{2}-(v \cdot w)^{2}-(u \cdot v)^{2}+2(u \cdot v)(u \cdot w)(v \cdot w)=((u \times v) \cdot w)^{2}
$$

which is surely identically satisfied, since each side equals the square of the volume of the parallelepiped of sides $u, v, w$.

\section{The main result}

Theorem 5.1. Assuming that the two-valued observables $A, B, C$ admit a quantum model, the following assertions $\sqrt{12}$ hold and are equivalent: i) $\hat{A}, \hat{B}$ satisfy the saturated Heisenberg-Schrödinger uncertainty relation for every state:

$$
\operatorname{Var}(\hat{A}) \operatorname{Var}(\hat{B})=\left(\frac{1}{2}\langle\{\hat{A}, \hat{B}\}\rangle-\langle\hat{A}\rangle\langle\hat{B}\rangle\right)^{2}+\left(\frac{1}{2 i}\langle[\hat{A}, \hat{B}]\rangle\right)^{2} ;
$$

ii) the following inequality 13 holds in each eigenstate $\psi_{k}(C)$ of $C(k=1,2)$ :

$$
\Delta A \Delta B \geq\left|\frac{1}{2}\langle\{\hat{A}, \hat{B}\}\rangle-\langle\hat{A}\rangle\langle\hat{B}\rangle\right| ;
$$

iii) the following inequality holds:

$$
4 p q r-(p+q+r-1)^{2} \geq 0 .
$$

Furthermore iii) implies the hypothesis and the previous inequalities are saturated if and only if $A, B, C$ admit a real quantum model.

Proof. i) follows from the hypothesis by lemma 4.4, since the operators associated to $A, B, C$ are self-adjoint, acting on a two-dimensional complex Hilbert space, by definition of quantum model. If i) holds for every state then, in particular, it shall hold in each of the two eigenstates of $C$; so, omitting the last addend and taking the square roots, one gets ii). By lemma 4.1, we can replace the operators

\footnotetext{
${ }^{12}$ Similar assertions hold taking any permutation of the operators $\hat{A}, \hat{B}, \hat{C}$.

${ }^{13}$ where $\Delta \hat{Z}:=\sqrt{\operatorname{Var}(\hat{Z})}$ denotes the standard deviation of $\hat{Z}$. The r. h. s. is said the correlation term.
} 
$\hat{A}, \hat{B}, \hat{C}$ with the corresponding spin operators $u_{A} \cdot \sigma, u_{B} \cdot \sigma, u_{C} \cdot \sigma$, achieving in each of the two eigenstates $\psi_{k}\left(u_{C}\right)$

$$
\Delta\left(u_{A} \cdot \sigma\right) \Delta\left(u_{B} \cdot \sigma\right) \geq\left|\frac{1}{2}\left\langle\left\{u_{A} \cdot \sigma, u_{B} \cdot \sigma\right\}\right\rangle-\left\langle u_{A} \cdot \sigma\right\rangle\left\langle u_{B} \cdot \sigma\right\rangle\right| ;
$$

so the difference between the squares of 1 . h. s. and the r. h. s. must satisfy

$$
\operatorname{Var}\left(u_{A} \cdot \sigma\right) \operatorname{Var}\left(u_{B} \cdot \sigma\right)-\left(\frac{1}{2}\left\langle\left\{u_{A} \cdot \sigma, u_{B} \cdot \sigma\right\}\right\rangle-\left\langle u_{A} \cdot \sigma\right\rangle\left\langle u_{B} \cdot \sigma\right\rangle\right)^{2} \geq 0
$$

that, taking account of (24) and (25) in the proof of lemma 4.4, can be written

$$
1-\left(u_{A} \cdot u_{B}\right)^{2}-\left(u_{B} \cdot u_{C}\right)^{2}-\left(u_{C} \cdot u_{A}\right)^{2}+2\left(u_{A} \cdot u_{B}\right)\left(u_{B} \cdot u_{C}\right)\left(u_{C} \cdot u_{A}\right) \geq 0
$$

namely, since $\cos \widehat{u_{A} u_{B}}=u_{A} \cdot u_{B}, \cos \widehat{u_{B} u_{C}}=u_{B} \cdot u_{C}, \widehat{u_{C} u_{A}}=u_{C} \cdot u_{A}$,

$$
1-\cos ^{2} \alpha-\cos ^{2} \beta-\cos ^{2} \gamma+2 \cos \alpha \cos \beta \cos \gamma \geq 0
$$

and this by iii) of theorem 2.2 implies the hypothesis. Further (32) is also equivalent to iii) because, with the notations of section 2 , its 1 . h. s. equals $144(4 p q r-$ $\left.(p+q+r-1)^{2}\right)$. Finally the saturation statement follows from the last part of theorem 2.2.

Remark 5.1. It appears quite astonishing that the, seemingly, much more weak relation in ii) above turns out rather to be equivalent to the Heisenberg-Schrödinger uncertainty relation in full generality.

\section{Ancillary results}

Corolary 6.1. Under the hypothesis of the preceding theorem, the observables $A, B, C$ admit a strictly complex quantum model, resp. a real quantum model, if and only i $\mathrm{ft}^{15}\langle[\hat{A}, \hat{B}]\rangle_{\psi_{k}(C)} \neq 0$, resp. $\langle[\hat{A}, \hat{B}]\rangle_{\psi_{k}(C)}=0$, in each eigenstate $\psi_{k}(C)$ of $C(k=1,2)$.

Proof. It has been shown in the proof of the preceding theorem that the assertion ii) is equivalent to the inequality (31), whose 1. h. s., taking account of (25), equals $\left(\left(u_{A} \times u_{B}\right) \cdot u_{C}\right)^{2}$ that, in turn, due to eq. (21) of lemma 4.2, equals $\left(\frac{1}{2 i}\left\langle\left[u_{A} \cdot \sigma, u_{B} \cdot \sigma\right]\right\rangle_{\psi_{1}\left(u_{C}\right)}\right)^{2}$. Therefore the assertion ii) of the theorem, thanks to lemma 4 , turns out to be equivalent to $\left(\langle[\hat{A}, \hat{B}]\rangle_{\psi_{k}(C)}\right)^{2} \geq 0$. Moreover it will be saturated as soon as (27) will be and this completes the proof.

\footnotetext{
${ }^{14}$ Cfr. Appendix, 4.

${ }^{15} \mathrm{Cfr}$. Note 12 . The symbol \langle\rangle$_{\psi}$ denotes the average computed in the state $\psi$.
} 
Corolary 6.2. If the observables $A, B, C$ admit a strictly complex quantum model then every couple of the associated operators do not commute. Further, for every state, at least two couples have non vanishing commutators averages.

Proof. Let $X, Y, Z$ be whichever permutation of $A, B, C$. The assumption $[\hat{X}, \hat{Y}]=$ 0 would imply $\langle[\hat{X}, \hat{Y}]\rangle_{\psi_{1}(Z)}=0$, against what asserted in the preceding corollary 6.1 , and this proves the first statement. Further, by lemma 4.4, for every state $\psi$ there is a unit vector $w \in S^{(2)}$ such that, due to eq. (21) of lemma 4.2, we can write

$$
\frac{1}{2 i}\left\langle\left[u_{X} \cdot \sigma, u_{Y} \cdot \sigma\right]\right\rangle_{\psi}=\left(u_{X} \times u_{Y}\right) \cdot w
$$

If this is zero then $w$ belongs to the plane spanned by $u_{X}, u_{Y}$ and may be neither in the plane $u_{Y}, u_{Z}$ nor in the plane $u_{Z}, u_{X}$, because $u_{A}, u_{B}, u_{C}$ are not coplanar by theorem 2.2. So we get $\frac{1}{2 i}\left\langle\left[u_{Y} \cdot \sigma, u_{Z} \cdot \sigma\right]\right\rangle_{\psi} \neq 0$. and $\frac{1}{2 i}\left\langle\left[u_{Z} \cdot \sigma, u_{X} \cdot \sigma\right]\right\rangle_{\psi} \neq 0$. and, by eq. (18) of lemma 4.1, the last statement is proven as well.

\section{Conclusions}

We have proven that the statistical invariant $4 p q r-(p+q+r-1)^{2} \geq 0$ is the expression of the Heisenberg-Schrödinger uncertainty relations for every couple of observables of the considered system. It depends neither on the values of the observables nor on their scales and units of measure but only on the transition probabilities and provide a condition not only necessary, but sufficient too, in order for a quantum model to exist. In particular the inequality is strict if and only if there exists a strictly complex quantum model. In this case some ancillary results involving commutators have been found. Furthermore real quantum models have been characterized by the saturation of the uncertainty relation ii) in theorem 5.1 or, alternatively, by the vanishing of the commutator average appearing in corollary 6.1 or, definitively, in terms of transition probabilities alone, by the equation $4 p q r-(p+q+r-1)^{2}=0$. The latter confirms however the exceptional character of real quantum models, requiring an unlikely functional dependence of the given transition probabilities. In closing, it is to highlight that, as the transition probabilities can be estimated starting from relative frequencies experimentally observed, we are able in principle, for the considered simple system, to deduce the mathematical quantum formalism from the Heisenberg uncertainty relations alone.

\footnotetext{
${ }^{16}$ Furthermore cfr. note 6.
} 


\section{References}

[1] Accardi L. and A. Fedullo (1982), On the statistical Meaning of Complex Numbers in Quantum Mechanics, Lettere al Nuovo Cimento 347 161-172.

[2] Accardi L. (1984), Some trends and problems in quantum probability, in Quantum Probability and applications to the quantum theory of irreversible processes, L. Accardi, A. Frigerio and V. Gorini (eds.), Springer LNM 1055 $1-19$.

[3] Klein F. (1893), Vergleichende Betrachtungen über neuere geometrische Forschungen, Mathematische Annalen 43 63-100. Gesammelte Abh., Springer, 1 (1921) 460-497). English translation: A comparative review of recent researches in geometry, by Mellen Haskell, Bull. N. Y. Math. Soc 2 (1892-1893) 215-249.

[4] Gudder S. and Zanghì N. (1982), Probability Models, Il Nuovo Cimento 79 B 2 291-301.

[5] Fedullo A. (1992), On the Existence of a Hilbert Space Model for Finite Valued Observables, Il Nuovo Cimento 107 B 12 1413-1426.

[6] Jauch J. M. (1968), Foundations of Quantum Mechanics, Addison-Wesley Publishing Company, 1968.

[7] von Neumann J. (1932), Mathematische Grundlagen der Quantenmechanik, Die Grundlehren der Mathematischen Wissenschaften, Band 38, Berlin, Springer. English translation: Mathematical Foundations of Quantum Mechanics, Princeton University Press, 1971.

[8] Heisenberg, W. (1927), Uber den anschaulichen Inhalt der quantentheoretischen Kinematik und Mechanik, Zeitschrift für Physik 43 (3-4) 172-198. English translation in [13, 62-84]

[9] Sen D. (2014), The uncertainty relations in quantum mechanics, Current Science 107 (2) 203-218.

[10] Robertson, H.P. (1929), The uncertainty principle, Physical Review 34 573574. Reprinted in [13, 127-128]

[11] Schrödinger, E. (1930), The uncertainty relations in quantum mechanics, Zum Heisenbergschen Unschärfeprinzip, Sitzungsberichte der Preussischen Akademie der Wissenschaften, Physikalisch-mathematische Klasse 14 296303. 
[12] Griffiths D. (2005), Quantum Mechanics, New Jersey, Pearson.

[13] Wheeler, J.A. and W.H. Zurek (eds) (1983), Quantum Theory and Measurement, Princeton N.J., Princeton University Press.

\section{Appendix}

1. Results quoted from [1].

Theorem 7. The following assertions are equivalent:

i) the transition matrices $P, Q, R$ admit a complex Hilbert space model;

ii) the transition matrices $P, Q, R$ admit a spin model;

iii) $\cos ^{2} \alpha+\cos ^{2} \beta+\cos ^{2} \gamma-1 \leq 2 \cos \alpha \cos \beta \cos \gamma$;

iv) $-1 \leq \frac{\cos ^{2} \frac{\alpha}{2}+\cos ^{2} \frac{\beta}{2}+\cos ^{2} \frac{\gamma}{2}-1}{2 \cos \frac{\alpha}{2} \cos \frac{\beta}{2} \cos \frac{\gamma}{2}} \leq 1$;

v) $-1 \leq \frac{p+q+r-1}{2 \sqrt{p q r}} \leq 1$

vi) $[\sqrt{p q}-\sqrt{(1-p)(1-q)}]^{2} \leq r \leq[\sqrt{p q}+\sqrt{(1-p)(1-q)}]^{2}$

Proposition 3 Tree vectors $a, b, c \in S^{(2)}$ satisfying

$$
\cos \alpha=\cos \widehat{a b}, \cos \beta=\cos \widehat{b c}, \cos \gamma=\cos \widehat{c a}
$$

exist if and only if

$$
\cos ^{2} \alpha+\cos ^{2} \beta+\cos ^{2} \gamma-1 \leq 2 \cos \alpha \cos \beta \cos \gamma
$$

Theorem 9. The transition matrices $P, Q, R$ admit a real Hilbert space model if and only if

$$
\begin{aligned}
& \frac{p+q+r-1}{2 \sqrt{p q r}}=+1 \text { or } \frac{p+q+r-1}{2 \sqrt{p q r}}=-1 \\
& \text { or equivalently } \\
& \sqrt{r}=\sqrt{p q}+\sqrt{(1-p)(1-q)} \text { or } \sqrt{r}=|\sqrt{p q}-\sqrt{(1-p)(1-q)}| .
\end{aligned}
$$

Theorem 10. The transition matrices $P, Q, R$ admit a real Hilbert space model if and only if they admit a spin model defined by a coplanar triple of vectors in $S^{(2)}$.

\section{Proof of lemma 4.1.}

Put for simplicity $z_{+}:=\frac{z_{1}+z_{2}}{2}$ and $z_{-}:=\frac{z_{1}-z_{2}}{2}$, by equation (10) we get

$$
\langle\hat{Z}\rangle=z_{+}+z_{-}\left\langle u_{Z} \cdot \sigma\right\rangle
$$

and, since $\left\langle\left(u_{Z} \cdot \sigma\right)^{2}\right\rangle=\langle\hat{1}\rangle=1$, 


$$
\left\langle\hat{Z}^{2}\right\rangle=\left(z^{2}\right)_{+}+2 z_{+} z_{-}\left\langle u_{Z} \cdot \sigma\right\rangle
$$

from which we obtain

$$
\operatorname{Var}(\hat{Z})=z_{-}^{2} \operatorname{Var}\left(u_{Z} \cdot \sigma\right) .
$$

Further, with easily understood notations, we have

$$
\frac{1}{2}\langle\{\hat{X}, \hat{Y}\}\rangle=x_{+} y_{+}+x_{-} y_{+}\left\langle u_{X} \cdot \sigma\right\rangle+x_{+} y_{-}\left\langle u_{Y} \cdot \sigma\right\rangle+\frac{1}{2}\left\langle\left\{u_{X} \cdot \sigma, u_{Y} \cdot \sigma\right\}\right\rangle,
$$

so that

$$
\frac{1}{2}\langle\{\hat{X}, \hat{Y}\}\rangle-\langle\hat{X}\rangle\langle\hat{Y}\rangle=x_{-} y_{-}\left(\frac{1}{2}\left\langle\left\{u_{X} \cdot \sigma, u_{Y} \cdot \sigma\right\}\right\rangle-\left\langle u_{X} \cdot \sigma\right\rangle\left\langle u_{Y} \cdot \sigma\right\rangle\right) ;
$$

last, quite directly, we get

$$
\frac{1}{2 i}\langle[\hat{X}, \hat{Y}]\rangle=x_{-} y_{-} \frac{1}{2 i}\left\langle\left[u_{X} \cdot \sigma, u_{Y} \cdot \sigma\right]\right\rangle
$$

3 Proof of lemma 4.2. By definition $u \cdot \sigma=\left[\begin{array}{cc}u_{3} & u_{1}-i u_{2} \\ u_{1}+i u_{2} & -u_{3}\end{array}\right]$, so that for every state $\psi$ one has

$$
\begin{gathered}
\langle u \cdot \sigma\rangle=\langle(u \cdot \sigma) \psi \mid \psi\rangle=\left[\overline{\psi_{1}} \quad \overline{\psi_{2}}\right]\left[u_{3} \psi_{1}+\left(u_{1}-i u_{2}\right) \psi_{2} \quad\left(u_{1}+i u_{2}\right) \psi_{1}-u_{3} \psi_{2}\right]^{T} \\
=2 u_{1} \Re\left(\overline{\psi_{1}} \psi_{2}\right)+2 u_{2} \Im\left(\overline{\psi_{1}} \psi_{2}\right)+u_{3}\left(\left|\psi_{1}\right|^{2}-\left|\psi_{2}\right|^{2 \cdot}\right) .
\end{gathered}
$$

Since ${ }^{17}$ as known $\psi_{1}(w)=\left[\begin{array}{ll}\sqrt{\frac{1+w_{3}}{2}} & \frac{w_{1}+i w_{2}}{\sqrt{2\left(1+w_{3}\right)}}\end{array}\right]^{T}$ and $\psi_{2}(w)=\left[\begin{array}{ll}\sqrt{\frac{1-w_{3}}{2}} & -\frac{w_{1}+i w_{2}}{\sqrt{2\left(1-w_{3}\right)}}\end{array}\right]^{T}$, putting them in the former formula, with easy calculations we get $\langle u \cdot \sigma\rangle_{\psi_{1}(w)}=$ $u \cdot w$ and $\langle u \cdot \sigma\rangle_{\psi_{2}(w)}=-u \cdot w$ as asserted in eq. (19). Further it is soon seen that $(u \cdot \sigma)^{2}=\hat{1}$, so that $\left\langle(u \cdot \sigma)^{2}\right\rangle=1$, for which, in the said states, $\operatorname{Var}(u \cdot \sigma)=\langle u \cdot \sigma\rangle^{2}-(\langle u \cdot \sigma\rangle)^{2}=1-(u \cdot w)^{2}$ as stated in eq. (20), that therefore is proven. Further, due te $\left[\sigma_{j}, \sigma_{k}\right]=2 i \varepsilon_{j k l} \sigma_{l}$ for every $j, k$, one has

\footnotetext{
${ }^{17}$ In a basis of $\mathcal{H}$ in which the Pauli matrices have the usual form (9), cfr. for example [1], p. 170.

${ }^{18} \varepsilon_{j k l}$ denotes the Levi-Civita symbol; on repeated indices summation is understood.
} 
$\frac{1}{2 i}[u \cdot \sigma, v \cdot \sigma]=\operatorname{Det}\left[\begin{array}{lll}\sigma_{1} & \sigma_{2} & \sigma_{3} \\ u_{1} & u_{2} & u_{3} \\ v_{1} & v_{2} & v_{3}\end{array}\right]=(u \times v) \cdot \sigma$, so that, taking the averages in the states $\psi_{k}(w)$ and considered that $\left\langle\sigma_{k}\right\rangle=w_{k}$ for $k=1,2,3,(21)$ is proven. Lastly, due to $\left\{\sigma_{h}, \sigma_{k}\right\}=2 \delta_{h k} \hat{1}$ for every $h, k$, we have $\{\sigma \cdot u, \sigma \cdot v\}=2 u \cdot v \hat{1}$ so that, taking the averages in whichever state, we get eq. (22) and the proof of the lemma is complete.

4. With the notations of section 2 , thanks to the trigonometric identity $\cos \theta=$ $2 \cos ^{2} \frac{\theta}{2}-1$, we can write $\cos \alpha=2 p-1, \cos \beta=2 q-1, \cos \gamma=2 r-1$, so that

$$
\begin{gathered}
1-\cos ^{2} \alpha-\cos ^{2} \beta-\cos ^{2} \gamma+2 \cos \alpha \cos \beta \cos \gamma= \\
1-(2 p-1)^{2}-(2 q-1)^{2}-(2 r-1)^{2}+2(2 p-1)(2 q-1)(2 r-1)
\end{gathered}
$$

that suitably simplified becomes $4\left(4 p q r-(p+q+r-1)^{2}\right)$ as asserted. 